\title{
ESTUDO DA SOLIDIFICAÇÃO/ESTABILIZAÇÃO DE UM RESÍDUO SÓLIDO INDUSTRIAL EM MATRIZES DE CIMENTO: AVALIAÇÃO DA LIXIVIABILIDADE E SOLUBILIDADE DE CONTAMINANTES
}

\author{
R. F. BERTOLI ${ }^{1}$, N. CAPUTO ${ }^{1}$, J. G. SGORLON ${ }^{2}$, M. C. S. GOMES ${ }^{1}$, M. L. MENEZES ${ }^{2}$ \\ ${ }^{1}$ Universidade Tecnológica Federal do Paraná, Processos Químicos \\ ${ }^{2}$ Universidade Tecnológica Federal do Paraná, Engenharia Química \\ E-mail para contato: renan_bertoli@hotmail.com
}

\begin{abstract}
RESUMO - O presente trabalho teve como objetivo a avaliação do comportamento de um resíduo sólido industrial na matriz cimentícia de blocos de concretos para pavimentação. O resíduo foi coletado, caracterizado quimicamente e utilizado na confecção dos blocos utilizando formulações com $2 \%$ a $10 \%$ de resíduo na massa cimentícia. Posteriormente, as peças foram submetidas a ensaios de lixiviação e solubilização para verificação da retenção de contaminantes. Os resultados obtidos mostraram que a incorporação do resíduo nos blocos de concreto não afetou as características de lixiviabilidade e solubilidade das peças, assim como houve a constatação de solidificação/estabilização dos metais na matriz de cimento.
\end{abstract}

\section{INTRODUÇÃO}

Resíduos industriais podem apresentar-se nas mais variadas formas, afetando locais muitas vezes distantes do local de sua geração (FRANCO, 2011). Paralelamente ao crescimento da geração desses resíduos amplia-se o anseio por um desenvolvimento sustentável. Uma alternativa para eliminação de elementos contaminantes ao ambiente, principalmente metais pesados são as técnicas de Solidificação/Estabilização ( $\mathrm{S} / \mathrm{E})$ de resíduos sólidos, um processo também conhecido como encapsulamento (encapsulação) ou fixação. Essa técnica pode ser implantada na reutilização de resíduos sólidos industriais que não possuem uma destinação adequada. É possível também proporcionar características positivas a outro material por meio de sua incorporação na matriz cimentícia, podendo assim ser utilizado na fabricação de artefatos de concreto com melhor qualidade para construção civil.

Neste contexto, o objetivo do presente trabalho foi a avaliação da solidificação/estabilização de um resíduo sólido industrial na matriz de cimento de blocos de concreto para pavimentação.

\section{MATERIAIS E MÉTODOS}




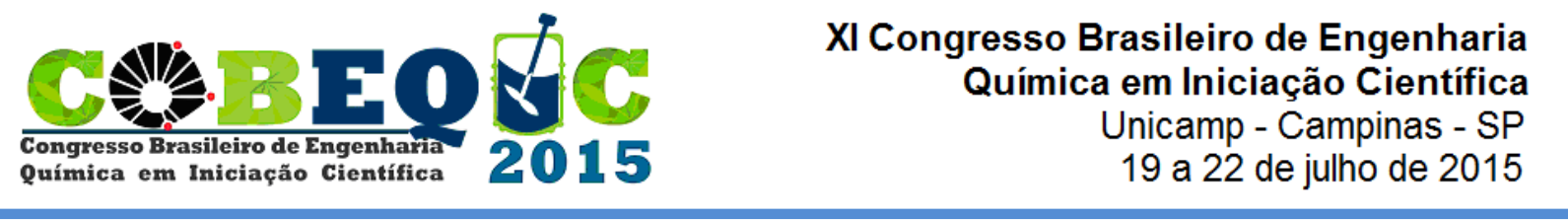

\subsection{Caracterização do Resíduo}

O resíduo utilizado foi coletado diretamente de uma indústria de galvanoplastia localizada na região de Piabiru - PR. Após coletado, o resíduo foi acondicionado em bombas plásticas devidamente lacradas e guardadas em local protegido.

Caracterização química: foram realizados por meio dos ensaios de lixiviação e solubilização segundo as normas ABNT NBR 10005 (2004) e ABNT NBR 10006 (2004), respectivamente. Para motivos de comparação também foram realizadas as análises de lixiviação e solubilização no cimento utilizado na fabricação dos blocos de concreto.

\subsection{Caracterizações dos Blocos de Concreto Fabricados}

A fabricação dos blocos de concreto com dimensões de $100 \mathrm{~mm}$ x $200 \mathrm{~mm}$ x $80 \mathrm{~mm}$ foi realizada na Fábrica de Artefatos da Universidade Estadual de Maringá. Os equipamentos necessários para a fabricação foram: equipamentos manuais (carriola e pás) para homogeneização e confecção da pasta de concreto e vibro-prensa semi-automática pneumática, para a moldagem dos blocos de concreto. $O$ traço utilizado para a fabricação do concreto dos blocos foi Franco (2011), que também estudou a incorporação de resíduos de galvanoplastia na fabricação de blocos de concreto para pavimentação.

Caracterização química: Os ensaios de lixiviação/solubilização fizeram parte da caracterização química dos blocos e foram realizados conforme as metodologias anteriormente citadas das normas ABNT NBR 10005 (2004) e ABNT NBR 10006 (2004), respectivamente, com detecção de contaminantes utilizando-se espectrômetro e absorção atômica e cromatógrafo de íons.

Cálculo da eficiência de retenção de contaminantes da matriz cimentícia: a determinação da eficiência de retenção dos metais na matriz de cimento (blocos) e foi calculada por meio da Equação 1:

$$
E_{\text {retenção }}=\frac{C_{\text {bruto }}-C_{\text {tratado }}}{C_{\text {bruto }}} \times 100
$$

Em que: $\mathrm{E}_{\text {retenção }}$ - Eficiência de retenção, em porcentagem (\%); $\mathrm{C}_{\text {bruto }}$ - Concentração do metal no extrato lixiviado/solubilizado do resíduo bruto, em $\left(\mathrm{mg} \mathrm{L}^{-1}\right) ; \mathrm{C}_{\text {tratado }}$ - Diferença entre a concentração do metal lixiviado/solubilizado nos blocos sem adição do resíduo (referência) e com adição do resíduo, em $\left(\mathrm{mg} \mathrm{L}^{-1}\right)$.

\section{RESULTADOS E DISCUSSÕES}

\subsection{Caracterização Química do Resíduo}


$\mathrm{Na}$ Tabela 1 encontram-se os contaminantes presentes no lixiviado do cimento CPV ARI e no resíduo utilizados na fabricação dos blocos de concreto, bem como os limites de concentração estabelecidos pela norma ABNT NBR 10004 (2004).

Tabela 1 - Concentração de contaminantes presentes no extrato lixiviado do cimento CPV ARI e do resíduo.

\begin{tabular}{c|c|c|c|c|c|c|c}
\hline Contaminante & $\begin{array}{c}\text { Limite NBR } \\
10004 / 2004 \\
\left(m g L^{-1}\right)\end{array}$ & $\begin{array}{c}\text { Cimento } \\
\left(m g L^{-1}\right)\end{array}$ & $\begin{array}{c}\text { Residuo } \\
\left(m g L^{-1}\right)\end{array}$ & Contaminante & $\begin{array}{c}\text { Limite NBR } \\
10004 / 2004 \\
\left(m g L^{-1}\right)\end{array}$ & $\begin{array}{c}\text { Cimento } \\
\left(m g L^{-1}\right)\end{array}$ & $\begin{array}{c}\text { Residuo } \\
\left(m g L^{-1}\right)\end{array}$ \\
\hline$B a$ & 70,00 & 25,111 & 3,981 & $H g$ & 0,10 & $<0,10$ & $<0,10$ \\
$C d$ & 0,50 & 0,0402 & 0,0865 & $A g$ & 5,00 & $<0,02$ & $<0,02$ \\
$P b$ & 1,00 & 0,801 & 0,217 & Fluoreto $\left(F^{-}\right)$ & 150 & n.d & 5,52 \\
$C r$ & 5,00 & 0,898 & 0,162 & & & \\
\hline
\end{tabular}

Os valores indicados por < (menor que) estão abaixo do limite de detecção do espectrômetro de absorção atômica; n.d: não detectado pelo cromatógrafo de íons.

Quando comparados com os valores estabelecidos pela norma vigente é possível observar que todos os contaminantes presentes tanto no resíduo como no cimento apresentaram concentrações lixiviadas abaixo dos limites máximos aceitáveis. Ou seja, isso permite classificar tanto o cimento quanto o resíduo industrial utilizado como resíduos sólidos Classe II, não perigosos.

Para a classificação dos resíduos quanto à sua natureza inerte (Classe IIA - não inerte ou Classe IIB - inerte), foi necessário a realização do ensaio de solubilização. Na Tabela 2 estão apresentadas as concentrações dos contaminantes solubilizados no cimento CPV - ARI e no resíduo industrial juntamente com os limites estipulados novamente pela norma $\mathrm{ABN}$ NBR 10004 (2004).

Tabela 2 - Concentração de contaminantes presentes no extrato solubilizado do cimento CPV - ARI e do resíduo.

\begin{tabular}{cccccccc}
\hline Contaminante & $\begin{array}{c}\text { Limite NBR } \\
10004 / 2004 \\
\left(m g L^{-1}\right)\end{array}$ & $\begin{array}{c}\text { Residuo } \\
\left(m g L^{-1}\right)\end{array}$ & $\begin{array}{c}\text { Cimento } \\
\left(m g L^{-1}\right)\end{array}$ & Contaminante & $\begin{array}{c}\text { Limite NBR } \\
10004 / 2004 \\
\left(m g L^{-1}\right)\end{array}$ & $\begin{array}{c}\text { Residuo } \\
\left(m g L^{-1}\right)\end{array}$ & $\begin{array}{c}\text { Cimento } \\
\left(m g L^{-1}\right)\end{array}$ \\
\hline$A l$ & 0,20 & 0,34 & $<0,30$ & $H g$ & 0,001 & $<0,10$ & $<0,10$ \\
$B a$ & 0,70 & 4,26 & 10,52 & $A g$ & 0,05 & $<0,02$ & $<0,02$ \\
$C d$ & 0,005 & 0,03 & 0,03 & $N a$ & 200 & 60,46 & 200,21 \\
$P b$ & 0,01 & $<0,10$ & 0,36 & Cloreto $\left(C l^{-}\right)$ & 250 & n.d & 1,02 \\
$C u$ & 2,00 & $<0,03$ & $<0,03$ & Fluoreto $\left(F^{-}\right)$ & 1,50 & n.d & 0,95 \\
$C r$ & 0,05 & 0,18 & 0,49 & Nitrato $\left(N O^{3-}\right)$ & 10,00 & n.d & 1,15 \\
$F e$ & 0,30 & 0,81 & 0,91 & Sulfato $\left(\mathrm{SO}_{4}{ }^{2-}\right)$ & 250 & n.d & n.d \\
$\mathrm{Mn}$ & 0,10 & 1,38 & 0,71 & & & & \\
\hline
\end{tabular}

Os valores indicados por < (menor que) estão abaixo do limite de detecção do espectrômetro de absorção atômica; n.d: não detectado pelo cromatógrafo de íons.

De acordo com os resultados pode-se observar que vários contaminantes apresentam valores superiores aos limites da norma brasileira. No resíduo industrial os contaminantes que estão em desacordo com a norma são: alumínio $(\mathrm{Al})$, bário $(\mathrm{Ba})$, cádmio $(\mathrm{Cd})$, cromo $(\mathrm{Cr})$, 
ferro $(\mathrm{Fe})$ e manganês $(\mathrm{Mn})$. No cimento são: bário $(\mathrm{Ba})$, cádmio $(\mathrm{Cd})$, chumbo $(\mathrm{Pb})$, cromo $(\mathrm{Cr})$, ferro $(\mathrm{Fe})$, manganês $(\mathrm{Mn})$ e sódio $(\mathrm{Na})$.

Com isso, verificar-se que ambos, cimento e resíduo industrial enquadram-se na classificação de resíduo sólido Classe II A - Não perigoso, não inerte, segunda a norma ABNT NBR 10004 (2004).

\subsection{Caracterização dos Blocos de Concreto Fabricados}

A concentração dos contaminantes presentes no extrato lixiviado dos blocos fabricados com o resíduo industrial, aos 28 dias de cura, estão listados na Tabela 3.

Tabela 3 - Concentração de contaminantes presentes no extrato lixiviado dos blocos fabricados com resíduo aos 28 dias de cura.

\begin{tabular}{cccccccc}
\hline Contaminante & $\begin{array}{c}\text { Limite NBR } \\
10004 / 2004 \\
\left(m g L^{-1}\right)\end{array}$ & Ref. & $2 \%$ & $3 \%$ & $4 \%$ & $5 \%$ & $10 \%$ \\
\hline$B a$ & 70,00 & 9,19 & 8,83 & 9,76 & 14,38 & 17,82 & 17,25 \\
$C d$ & 0,50 & 0,05 & 0,03 & 0,07 & 0,04 & 0,03 & 0,04 \\
$P b$ & 1,00 & 0,36 & 0,36 & 0,28 & 0,41 & 0,29 & 0,51 \\
$C r$ & 5,00 & 0,15 & 0,17 & 0,18 & 0,21 & 0,21 & 0,24 \\
$H g$ & 0,10 & $<0,10$ & $<0,10$ & $<0,10$ & $<0,10$ & $<0,10$ & $<0,10$ \\
$A g$ & 5,00 & 0,03 & $<0,02$ & $<0,02$ & $<0,02$ & $<0,02$ & $<0,02$ \\
Fluoreto $\left(F^{-}\right)$ & 150 & n.d & n.d & n.d & n.d & n.d & n.d \\
\hline
\end{tabular}

Os valores indicados por < (menor que) estão abaixo do limite de detecção do aparelho utilizado n.d: não detectado pelo cromatógrafo de íons.

Por meio dos resultados apresentados pode-se observar que em todas as porcentagens de substituição de agregado miúdo por resíduo, as concentrações de contaminantes não ultrapassaram os limites impostos pela legislação. Ainda com base nos valores apresentados na Tabela 3, pode-se verificar que o resíduo sólido proveniente dos blocos fabricados, após sua utilização, pode ser classificado como Classe II - não perigoso.

$\mathrm{Na}$ Tabela 4 são apresentados os contaminantes presentes no extrato solubilizado dos blocos fabricados com o resíduo industrial aos 28 dias de cura. Como as concentrações de chumbo $(\mathrm{Pb})$ e mercúrio $(\mathrm{Hg})$ ficaram abaixo do limite de detecção do aparelho, os mesmos não foram considerados na tabela.

As concentrações dos elementos alumínio $(\mathrm{Al})$, bário $(\mathrm{Ba})$, cádmio $(\mathrm{Cd})$, cromo $(\mathrm{Cr})$ e manganês $(\mathrm{Mn})$ obtidas no extrato solubilizado dos blocos fabricados, atingiram valores acima do permitido pela norma ABNT NBR 10004 (2004), com isso o resíduo dos blocos pode ser classificado como Classe IIA, não perigoso, não inerte. Na incorporação de $10 \%$ de resíduo, observando as concentrações dos contaminantes, é possível verificar que não houve favorecimento na solubilização dos contaminantes dos blocos fabricados. 
Tabela 4 - Concentração de contaminantes presentes no extrato solubilizado dos blocos fabricados com resíduo aos 28 dias de cura.

\begin{tabular}{|c|c|c|c|c|c|c|c|}
\hline Contaminante & $\begin{array}{c}\text { Limite NBR } \\
10004 / 2004 \\
\left(\mathrm{mgL}^{-1}\right)\end{array}$ & Ref. & $2 \%$ & $3 \%$ & $4 \%$ & $5 \%$ & $10 \%$ \\
\hline$A l$ & 0,20 & 0,91 & 0,65 & 0,75 & 0,35 & 0,26 & 0,17 \\
\hline$B a$ & 0,70 & 8,30 & 10,92 & 13,60 & 9,45 & 12,67 & 15,69 \\
\hline$C d$ & 0,005 & 0,04 & 0,04 & 0,06 & 0,05 & 0,05 & 0,042 \\
\hline $\mathrm{Cu}$ & 2,00 & $<0,03$ & $<0,03$ & $<0,03$ & $<0,03$ & $<0,03$ & $<0,03$ \\
\hline $\mathrm{Cr}$ & 0,05 & 0,19 & 0,15 & 0,19 & 0,16 & 0,22 & 0,20 \\
\hline $\mathrm{Fe}$ & 0,30 & $<0,06$ & $<0,06$ & $<0,06$ & $<0,06$ & $<0,06$ & $<0,06$ \\
\hline$M n$ & 0,10 & 0,85 & 0,74 & 0,77 & 1,05 & 0,98 & 0,91 \\
\hline$A g$ & 0,05 & $<0,02$ & $<0,02$ & $<0,02$ & $<0,02$ & $<0,02$ & $<0,02$ \\
\hline $\mathrm{Na}$ & 200 & 40,21 & 40,39 & 40,34 & 40,49 & 43,01 & 41,33 \\
\hline Cloreto $\left(\mathrm{Cl}^{-}\right)$ & 250 & 1,15 & 1,30 & 1,42 & n.d & 1,39 & n.d \\
\hline Fluoreto $\left(F^{-}\right)$ & 1,50 & 0,26 & 0,25 & 0,35 & 0,18 & 0,27 & 0,19 \\
\hline $\operatorname{Nitrato}\left(\mathrm{NO}_{3}^{-}\right)$ & 10,00 & 5,37 & 2,90 & 1,96 & 2,74 & 3,78 & 6,82 \\
\hline Sulfato $\left(\mathrm{SO}_{4}^{2-}\right)$ & 250 & 30,26 & 30,45 & 54,02 & 39,33 & 35,54 & 30,63 \\
\hline
\end{tabular}

Os valores indicados por < (menor que) estão abaixo do limite de detecção do aparelho utilizado.

A Tabela 5 contém os resultados da eficiência de retenção de metais via lixiviado dos blocos fabricados.

Tabela 5 - Resultados da eficiência de retenção de metais via lixiviado.

\begin{tabular}{cccccc}
\hline & \multicolumn{5}{c}{ Eficiência de retenção (\%) } \\
Contaminantes & $2 \%$ & $3 \%$ & $4 \%$ & $5 \%$ & $10 \%$ \\
\hline$B a$ & 108,99 & 85,78 & $-30,39$ & $-116,88$ & $-102,41$ \\
$C d$ & 117,92 & 71,91 & 104,28 & 114,68 & 111,56 \\
$P b$ & 101,84 & 136,87 & 80,64 & 132,72 & 30,87 \\
$C r$ & 83,33 & 78,09 & 58,33 & 58,33 & 39,81 \\
\hline
\end{tabular}

O cádmio $(\mathrm{Cd})$, chumbo $(\mathrm{Pb})$ e o cromo $(\mathrm{Cr})$ valores de eficiência de retenção, dando maior destaque ao cádmio, pois sua retenção foi total. A prata $(\mathrm{Ag})$ e o mercúrio $(\mathrm{Hg})$ não participaram do cálculo de eficiência, pois suas concentrações no extrato lixiviado do resíduo e do cimento (Tabela 1), estiveram abaixo do limite de detecção. O bário (Ba) comportou-se de forma muito negativa, pois sua eficiência de retenção aos $4 \%, 5 \%$ e $10 \%$ de incorporação fora de $-30,39 \%,-116,88$ e $-102,41 \%$, respectivamente, pois no extrato solubilizado e lixiviado do cimento já existe grande concentração deste contaminante, causando interferências na eficiência de retenção.

Segundo Conner (1990), quando se utiliza cimento Portland para S/E resíduos sólidos industriais, o $\mathrm{pH}$ da matriz favorece a conversão de cátions em hidróxidos, óxidos e carbonatos insolúveis, incorporando íons metálicos à estrutura cristalina da matriz de cimento. Esse fato pode estar diretamente relacionado a alta eficiência de retenção que a maioria dos metais estudados apresentou na Tabela 6 . 
Tabela 6 - Resultados da eficiência de retenção do extrato solubilizados dos blocos fabricados.

\begin{tabular}{cccccc}
\hline & \multicolumn{5}{c}{ Eficiência de retenção (\%) } \\
Contaminantes & $2 \%$ & $3 \%$ & $4 \%$ & $5 \%$ & $10 \%$ \\
\hline$A l$ & 175,30 & 147,02 & 264,28 & 293,15 & 317,86 \\
$B a$ & 38,54 & $-24,40$ & 73,01 & $-2,42$ & $-73,39$ \\
$C d$ & 123,03 & 58,48 & 73,33 & 75,76 & 106,06 \\
$C r$ & 118,71 & 99,72 & 115,64 & 83,52 & 92,46 \\
$M n$ & 108,10 & 105,93 & 85,53 & 90,52 & 95,73 \\
$N a$ & 99,69 & 99,78 & 99,52 & 95,36 & 98,14 \\
\hline
\end{tabular}

Via extrato solubilizado, a maioria dos metais apresentou alta eficiência de retenção. $\mathrm{O}$ alumínio $(\mathrm{Al})$ e cádmio $(\mathrm{Cd})$ apresentaram retenções superiores a $100 \%$, já o cromo $(\mathrm{Cr})$, manganês $(\mathrm{Mn})$ e sódio $(\mathrm{Na})$ apresentaram aproximadamente $100 \%$ retenção. O bário $(\mathrm{Ba})$ novamente obteve eficiência de retenção negativa, devido sua grande concentração no lixiviado e solubilizado do cimento.

\section{CONCLUSÃO}

O resíduo utilizado neste trabalho foi classificado segundo a norma ABNT NBR 10004 (2004) como não perigoso e não inerte (Classe II-A). Assim há necessidade de acompanhamento especial para o descarte final do mesmo. No entanto, pode-se concluir que a incorporação de até $10 \%$ de resíduo industrial no concreto das peças para pavimentação não afetou a lixiviabilidade, nem a solubilidade de contaminantes, bem como, contatou-se a estabilização/solidificação da maioria dos metais na matriz cimentícia.

\section{REFERENCIAS}

. NBR 10004. Resíduos Sólidos - Classificação. Rio de Janeiro, 2004.

. NBR 10005. Procedimento para Obtenção de Extrato Lixiviado de Resíduos Sólidos. Rio de Janeiro, 2004.

. NBR 10006. Procedimento para Obtenção de Extrato Solubilizado de Resíduos Sólidos. Rio de Janeiro, 2004.

CONNER, J.R. Chemical fixation and solidification of hazardous wastes. New York: Van Nostrand Reinhold, 1990.

FRANCO, J. M. Produção de blocos de concreto para pavimentação (BCP) utilizando resíduos de galvanoplastia. Dissertação - Pós-Graduação em Engenharia Urbana, Universidade Estadual de Maringá, Maringá, 2011. 